\title{
Simulated Optical Properties of Gold Nanocubes and Nanobars by Discrete Dipole Approximation
}

\author{
Mohammed Alsawafta, Mamoun Wahbeh, and Vo-Van Truong \\ Department of Physics, Concordia University, Montréal, QC, Canada H4B 1 R6 \\ Correspondence should be addressed to Vo-Van Truong, tvovan@alcor.concordia.ca \\ Received 6 July 2011; Accepted 4 October 2011 \\ Academic Editor: Sherine Obare
}

Copyright () 2012 Mohammed Alsawafta et al. This is an open access article distributed under the Creative Commons Attribution License, which permits unrestricted use, distribution, and reproduction in any medium, provided the original work is properly cited.

\begin{abstract}
The absorption spectra for a gold nanocube and for a gold nanobar are calculated by using the Discrete Dipole Approximation (DDA). The results show the excitation of a single albeit broad surface plasmon (SP) band of the gold nanocube. The extinction cross section of the gold nanocube is dominated by the absorption cross section that gains importance as the width increases. Further increasing the nanocube size beyond $80 \mathrm{~nm}$ will result in an optical response mainly characterized by scattering properties. The absorption spectrum of the nanobar shows the excitation of both the longitudinal mode (LM) and the transverse mode (TM). The nanobar is also compared to a cylinder, a spherically capped cylinder, and a spheroid of the same aspect ratio. The band position of the TM of the nanobar is red-shifted as compared to the ones calculated for other morphologies, while the LM is either blue-shifted or red-shifted depending on the morphologies considered.
\end{abstract}

\section{Introduction}

Noble metal nanoparticles of a size smaller than the wavelength of the incident radiation can effectively scatter and selectively absorb light at a certain wavelength in the visible and near-infrared regions. Faraday [1] was the first to notice such optical properties of the metallic nanoparticles. Due to the interaction of the incident electromagnetic radiation with the metallic nanoparticles, the conduction electrons exhibit collective oscillations parallel to the incident electric field, resulting in the redistribution of the polarization charges at the metal/dielectric interface. The collective oscillatory motion of electrons at the metal surface is known as Surface Plasmon Resonance (SPR) [2-4]. If the electronic motion is confined locally around the nanoparticles, the oscillation is called Localized Surface Plasmon Resonance (LSPR). The LSPR is responsible for the fascinating optical properties of the metal at the nanometric scale as compared to its bulk counterpart [5]. The optical characteristics of the LSRP (the frequency and the bandwidth) are strongly dependent on the size, the distribution of the polarization charges over the surface area of the nanoparticles (the shape), the dielectric function of the surrounding material, the composition of the nanostructure, and the coupling between the plasmon resonances in nearby structures [6-13]. In addition, the optical response of the nanostructures depends on the frequency and the polarization state of the incident radiation [11]. The optical responses of the nanostructures have been studied extensively both experimentally [14-20] and theoretically [21-25].

The metallic nanoparticles have thus attracted intensive interest from researchers because of the tremendous potential applications in many fields including optical imaging and photothermal treatment [26-29], Surface-Enhanced Raman Scattering (SERS) [30-32], chemical and biological sensing [33-35], photothermic transducer of laser radiation [36], nanoantenna [37], and solar energy conversion technology [38-40]. Due to the high symmetry order of the spherical nanoparticles, it is expected that the absorption spectrum exhibits a single dipolar plasmonic band. Changing the morphology of the nanoparticle resulted in changing the symmetry order, which has a dramatic effect on the distribution of the polarization charges over the surface area of the nanostructure. The redistribution of the charges results in tuning 
the frequency and the type of the excited plasmon mode, namely, the TM, the LM, and the high-order multipole resonance modes [41-43].

Tailoring the optical properties of the metallic nanostructures of different size and shape is a desired aim for technological applications. Simulation of the optical response of the metallic nanoparticles is considered as the first step to achieve this goal. Many computational tools have emerged to model the optical properties of these nanostructures. Based on the analytical solution of Maxwell's equation, the Discrete Dipole Approximation (DDA) [44-49] is a powerful technique to calculate the absorption and scattering cross sections of nanostructures of arbitrary shape, structure, and composition. It can be used for a single particle and for assemblies of particles that are surrounded by a medium with a complex dielectric function. Many research groups used DDA to simulate the optical properties of metallic nanostructures of different shapes and sizes (nanospheres [10,13], nanorods [22, 23, 50], and nanoprism [41]). Among the interesting nanostructures, one can cite the nanocube and the nanobar. Updated nanofabrication techniques are indeed able to produce a well-defined nanocube [51-57] or nanobar [57] of different size distributions and even an ordered 2-D array of different configurations [58, 59]. It would thus be of great interest to examine theoretically in detail the properties of these particles. This is precisely the objective of the present study as the DDA is employed to simulate the absorption spectrum for both an isolated gold nanocube and an isolated nanobar. For the nanocube, the main contribution to the extinction cross section from either the absorption or the scattering is discussed. The results found will be useful for determining the spatial deposition of gold nanocubes in the designing of, for example, plasmonic solar cells. The absorption properties of the nanorod will be investigated in terms of the length distribution and the relative orientation to the incident light. The absorption of this latter nanostructure will be compared with the ones calculated for other nanoparticles which exhibit two-fold symmetry.

This study is organized as follows. In Section 2, we discuss briefly the basic idea of the computational tool DDA, followed by a presentation of the target geometry, the corresponding structural parameters and the relative orientation of the nanostructure in the incident electromagnetic field. Section 3 will include the results and discussion, which is divided into two subsections. The first subsection concerns the discussion on the modeled absorption spectra of a single nanocube. The absorption efficiency will be compared with the one for a spherical particle of the same size and volume. The second subsection studies the changes in the optical properties of the nanocube due to elongation of the structure along one of its axis, resulting in an evolution of the nanobar. The optical response of the nanobar is then compared with the one of the plain cylinder, a spherically capped cylinder, and an ellipsoid of the same aspect ratio (AR).

\section{Discrete Dipole Approximation (DDA)}

The exact solution of Maxwell's equation for some specific geometries is known, namely, in the case of the spherical particle, the infinite cylinder, and the spheroid $[60,61]$. For other morphologies, an approximated solution is often required. DDA indeed offers a powerful numerical tool to model the optical properties of nanostructures of arbitrary shape, structure, and composition. The description of the mathematical formulation of the DDA is out of the scope of this paper, but more details can be found in the references cited. Briefly, in the response of the incident electromagnetic field, the polarization charges of the metallic nanoparticles are redistributed. Therefore, the electron cloud is shifted relative to the positive core resulting in an induced electric dipole moment. Based on the interaction between the induced dipoles, the optical response of the metallic nanostructures can be calculated. The DDA requires breaking up the target under investigation into three-dimensional dipoles. The induced dipoles are sitting on a periodic square lattice, such that the side length of one lattice unit represents the dipole separation $(d)$. To provide an accurate description of the target's geometry, a large number of the dipoles are required to mimic properly the structural parameters of the nanostructures. The description of the actual volume $(V)$ of the target solid material depends on the number of dipoles and the size of the square unit lattice $\left(d^{3}\right)$. The size of the target is also characterized by an equal volume sphere of effective radius $a_{\text {eff }}=(3 v / 4 \pi)^{1 / 3}$. The desired output of the DDA in this study is the absorption cross section $(C)$ of the nanostructure normalized to its geometrical cross section $\left(\pi a_{\text {eff }}^{2}\right)$, which yields the corresponding efficiencies $Q=C / \pi a_{\text {eff. }}^{2}$. The open-source Fortran-90 software package (DDSCAT 7.1) was used to calculate the absorption cross section.

\section{The Structural Parameters and the Morphology of the Target}

To study the effect of the various parameters such as the size and the angle of the incidence on the optical characteristics of LSPR of the metallic nanocube and nanobar, batches of simulations were performed. The structural parameter of the nanocube is determined by the side length as shown in Figure 1(a). Scanning Electron Microcopy (SEM) images for experimentally prepared particles showed that the width of the nanocubes ranged between $20-100 \mathrm{~nm}$ [51-59]. Since the nanocube is considered as seeds for the synthesis of the nanobar by stretching the former along one of its axis, the width of the nanobar cross section will still be represented by the side length of the preliminary seeds. The structural parameter of the nanobar is defined further by the aspect ratio (the actual length $(L)$ divided by the width $(W)$ ) as illustrated in Figure 1(b).

It is assumed that the incident light is linearly polarized in the $y$-direction, and the direction of the propagation is considered along the $x$-axis. The orientation of the target is achieved by rotating the major axis with respect to the propagation direction by an angle $\theta$ as shown in Figure 1(c). For modeling of the optical response of the nanostructure under investigation, the bulk gold dielectric function has been extracted from Johnson and Christy [62]. 


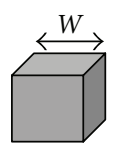

(a)

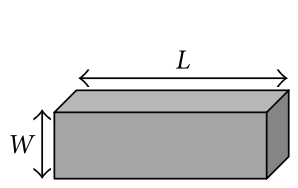

(b)

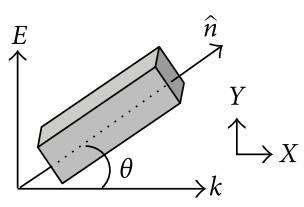

(c)
Figure 1: Structural parameters of (a) a cube, (b) a nanobar, (c) orientation of the nanobar with respect to the incident radiation (p-polarized light).

The effective radius of the equivolume sphere for the nanocube and the nanobar is $r_{\text {eff }}=(3 / 4 \pi)^{1 / 3} W$ and $r_{\text {eff }}=$ $\left(3 W^{2} L / 4 \pi\right)^{1 / 3}$, respectively.

\section{Results and Discussion}

Theoretical calculations of the absorption spectra are done by using DDA. All the calculations presented here refer to the air as the surrounding material where the nanostructures are embedded. Since the size of the nanoparticle considered here is larger than the mean free path of the conduction electrons, the scattering process of the conduction electron on the nanoparticle surface has insignificant effect on the dielectric function, and no further size correction is required for the bulk dielectric function. Mainly, the discussion is divided into two main parts with the first part showing the dependency of the absorption spectrum of an isolated gold nanocube on the size. A comparison between a gold nanocube and a silver nanocube of the same size will be presented. The evolution of the nanobar from the nanocubic seeds will be the topic of the second subsection. The effect of the orientation and the length distribution on the absorption efficiency of the gold nanobar will be discussed in terms of the band position and the intensity of plasmonic mode.

4.1. The Optical Properties of an Isolated Nanocube. To investigate the size effect on the optical behaviour of an isolated metallic nanocube, a series of absorption spectra have been simulated.

The side length of the nanocube has been chosen in the range $20-100 \mathrm{~nm}$. In all the simulations, the interdipole separation was kept constant at around $1 \mathrm{~nm}$, giving a total of 8000 to 1000000 dipoles per nanocube. The chosen number of dipoles was sufficient to mimic the structure of the nanocube properly and to achieve a proper convergence of the simulation.

The result of the simulations shows that the absorption spectrum depends strongly on the size of the nanocube. Upon increasing the size, it is found that the amplitude increases linearly. With increasing $W$, the extinction efficiency has mainly contributions from the scattering cross section resulting in a decreased absorption amplitude, that is, when $W>80 \mathrm{~nm}$ as shown in Figure 2(a). The dependency of the absorption amplitude on the nanocube size is illustrated in Figure 2(b) and it fits with a Gaussian function. In addition, the band position of LSPR band is affected by changing the width, and it appears linearly redshifted with increasing side width as shown in Figure 2(c). The observed linear relation is in good agreement with published experimental results in the case of the silver nanocube [63]. The calculated absorption spectrum of a nanocube with a width of $45 \mathrm{~nm}$ is compared with the one measured recently by $\mathrm{Wu}$ et al. for gold nanocubes in water [64]. The band position of the calculated spectrum is at a longer wavelength as compared to the observed experimental one. The difference in the wavelength locations between the experimental and calculated bands would be mainly attributed to the sharp edge nanocube considered in the simulation. The synthesized nanocubes have rounded edges which would result in a blue shift due to decrease in the particle size. Experimental observations of this nature were also made regarding triangular silver nanoparticles with sharp and rounded edges [65].

The optical response-size dependency can be used effectively in Plasmonic Thin Film Solar Cells (PTFSC) by depositing the nanocubes with $W>80$ on the top of the solar cell to enhance the light scattering. They act like antenna to direct the scattered light into the interior part of the cell for more absorption. The smaller ones ( $W \leq 80 \mathrm{~nm}$ ) could be deposited between the active layers to increase the photogeneration of the electron-hole pairs and hence improve the light trapping capacity of the device due to the enhancement of the near electromagnetic field around the nanoparticles [66].

The absorption spectrum of the metallic nanocube of side length $50 \mathrm{~nm}$ is compared with that of two different spheres as shown in Figure 3(a); the first one is of size $50 \mathrm{~nm}$ (diameter) and the other has an equal volume to the nanocube. The calculated spectra of the nanocube show the excitation of a single plasmonic mode as in the case of the sphere. The polarization charges redistribution over a larger surface area of the nanocube as compared with the two spheres resulted in enhances in the absorption intensity. The nanocube has several symmetry axes, thus it is expected that the absorption spectrum exhibits several plasmonic bands. It seems however that the band positions of those modes are very close to each other making them indistinguishable, resulting in the excitation of a single broadened band. The LSPR band is red-shifted as compared to the corresponding spheres. In the case of the silver nanocube, the plasmonic bands which correspond to the multifold symmetry are well separated, resulting in the excitation of more than a single band as shown in Figure 3(b). It is well known that the polarization charges accumulated at a sharper area like the corner of the nanocube will result in an increase in the separation between the electron cloud and the positive core, and thus the electrostatic columbic force (restoring force) is decreased. This increasing separation will lead to a red shift of the observed plasmonic band as compared to the absorption spectrum for a corresponding spherical particle. The optical response of the nanocube is not affected by its orientation relative with respect to the incident electric field.

4.2. The Optical Properties of an Isolated Nanobar. As mentioned earlier, the morphology of the nanobar evolves from 


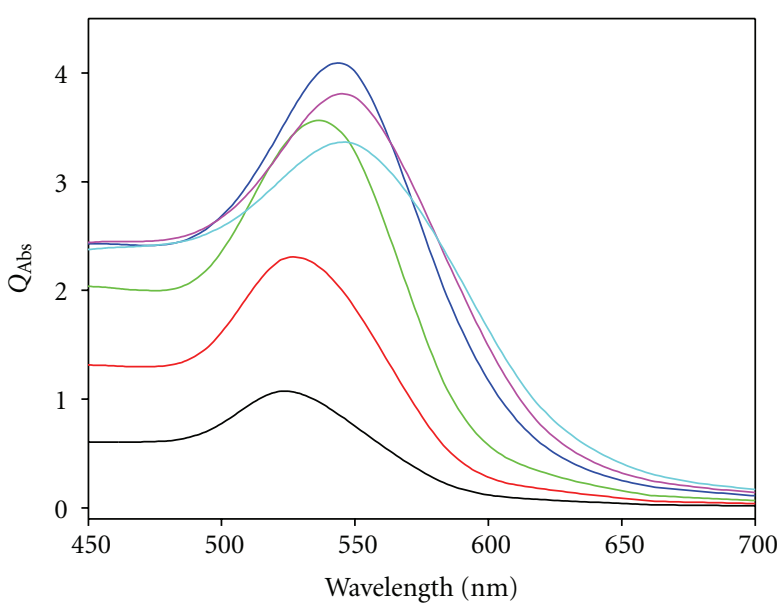

$W(\mathrm{~nm})$

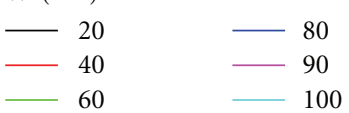

(a)

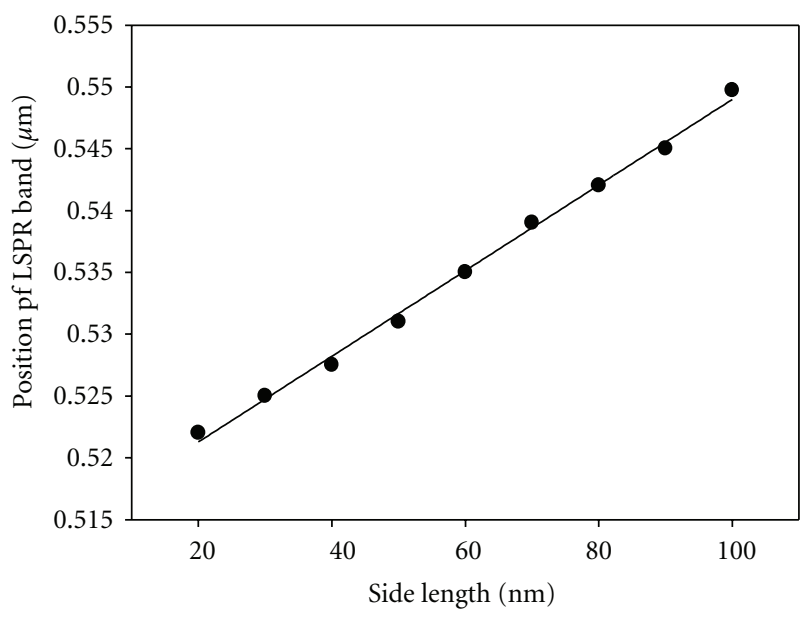

(c)

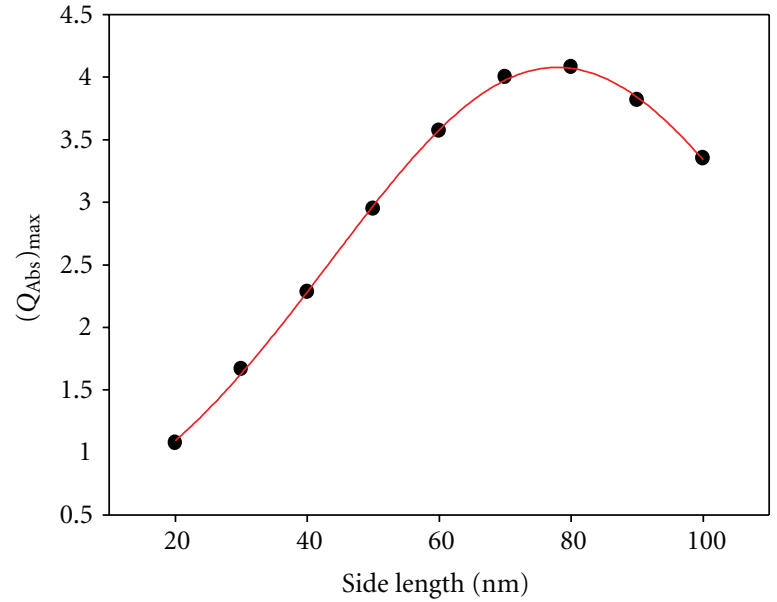

(b)

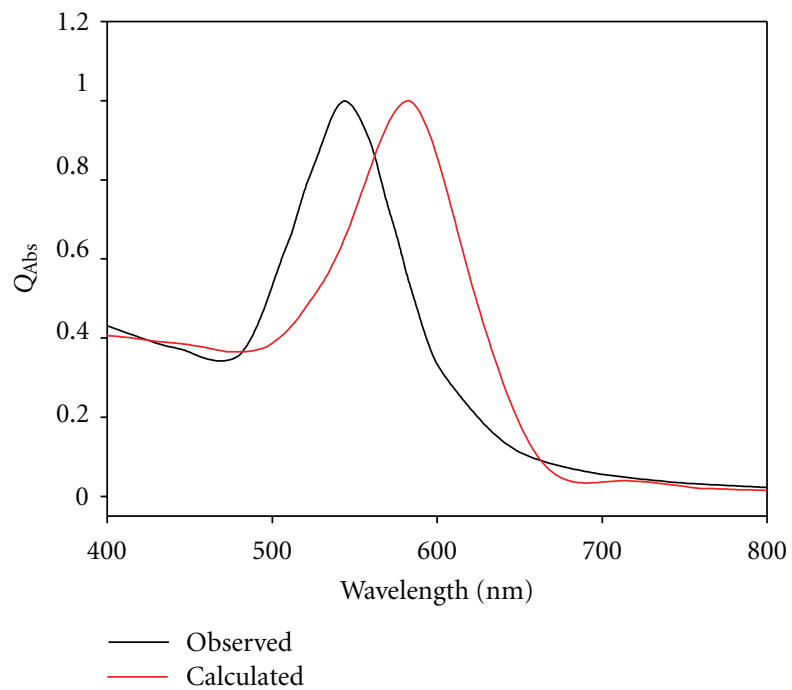

(d)

Figure 2: (a) Dependency of the absorption spectrum on the nanocube width, (b) the maximum absorption efficiency as a function of the width, (c) position of the LSPR band versus the nanocube width, and (d) comparison between observed and calculated absorption spectra of a gold nanocube of width $45 \mathrm{~nm}$.

the preliminary nanocube seed by elongation along one of its axis, and the cross section of the nanobar is still represented by the side length. As the morphology of the cube nanoparticle is changed to that of an elongated nanobar, the single plasmon band splits into two bands, the longitudinal band and the transverse band that are, respectively, due to oscillations of the polarization charges along and perpendicular to the major axis of the nanobar. The splitting occurs at a specific incident angle of the incident p-polarized light. The electric field of the p-polarized light has two components, respectively, along and perpendicular to the main axis of the nanobar. The s-polarized light has an electric field component perpendicular to the main axis of the nanobar, leading to the excitation of the transverse mode, and the longitudinal mode is not observed even at different incident angles. Figure 4 shows the absorption of a nanobar of width $40 \mathrm{~nm}$ and length $80 \mathrm{~nm}$. The intensity of LM and TM modes depends strongly on the incident angle. The TM is inversely related to the angle, while the LM is directly proportional to it. The band positions of both modes remain at the same wavelength. At the two extremes of the incident angles $\left(0^{\circ}\right.$, $90^{\circ}$ ), only one plasmon band is observed due to oscillations of the polarization charges parallel or perpendicular to the main axis. Both modes contribute to the total absorption spectra at intermediate angles in the range from $15^{\circ}$ to $75^{\circ}$. 


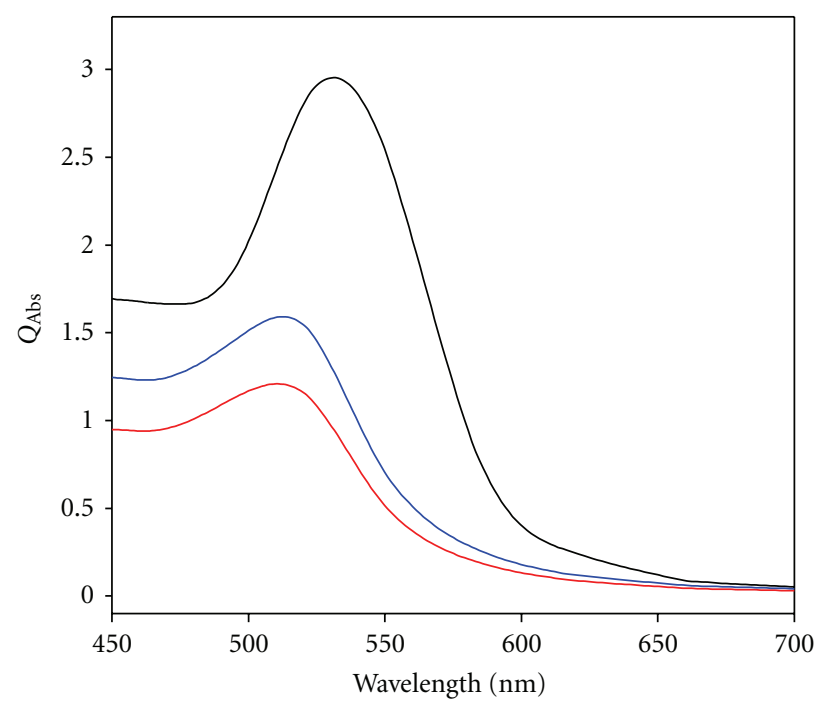

(a)

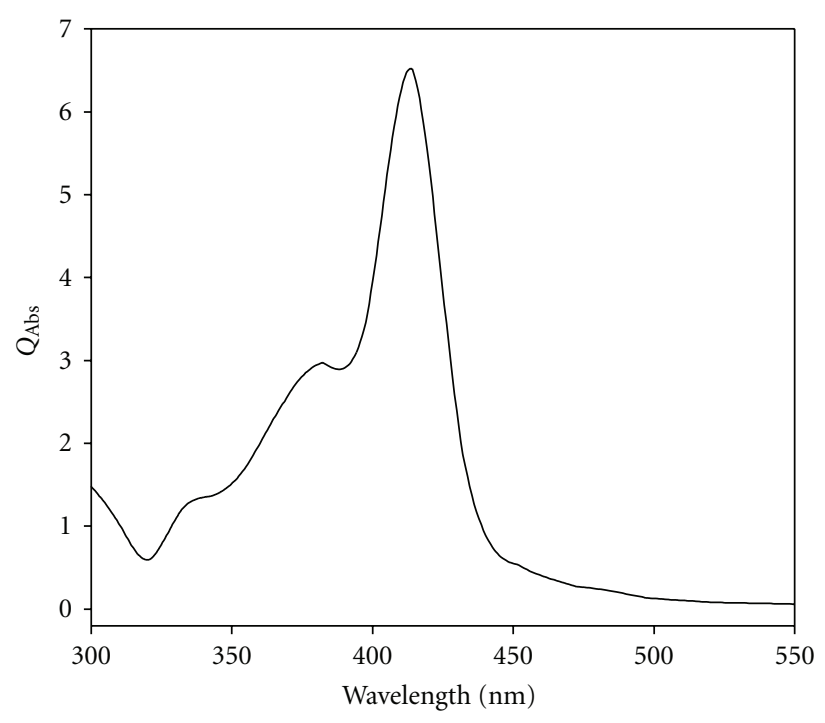

(b)

Figure 3: The calculated absorption spectrum for (a) a gold nanocube of width $50 \mathrm{~nm}$ (black), a sphere of diameter $50 \mathrm{~nm}$ (red), and a sphere that has the same volume as the nanocube (blue), (b) a silver nanocube of width $50 \mathrm{~nm}$.

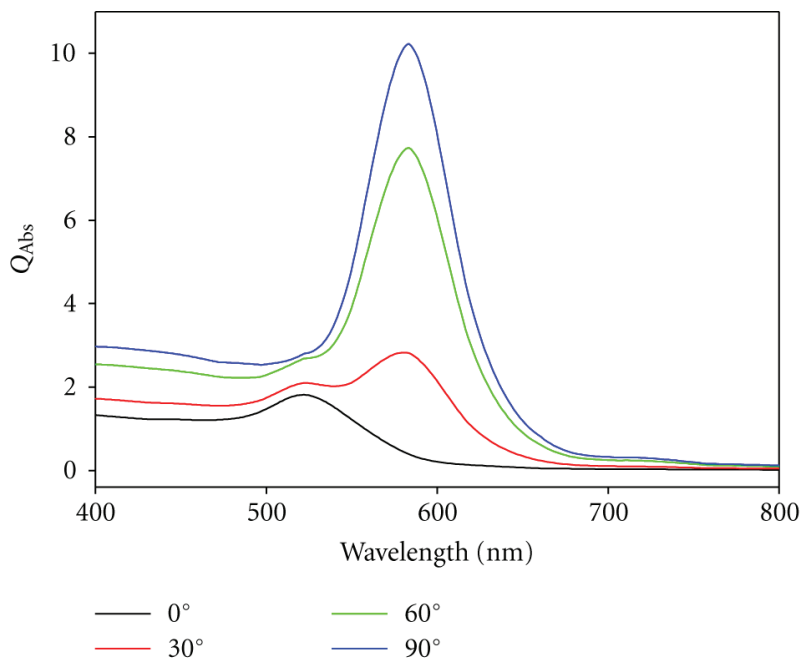

FIGURE 4: Absorption spectra for a gold nanobar $(40 \times 80 \mathrm{~nm})$ at different incident angles and p-polarized light.

To investigate the effect of the nanobar length on the absorption spectrum, a series of simulation were performed for different lengths. The calculations were performed at a constant width $(40 \mathrm{~nm})$ with various lengths $L \in\{60$, $80,100,120,140\} \mathrm{nm}$ and the corresponding $\mathrm{AR} \in\{1.5$, $2,2.5,3,3.5\}$. Figure $5(a)$ shows the calculated spectra at various $\mathrm{AR}$ at $\theta=30^{\circ}$. It can be seen that the plasmon band of the nanocube splits into two modes as the length increases. The position of the longitudinal band is observed to be the major change in the optical response of the nanobar due to the change in the structural parameters, whereas an insignificant change in the band position of the TM has been observed upon the increase in length. The band position can therefore be tuned in both the visible and near infrared regions. The change in the longitudinal band position with aspect ratio shows a linear variation. The linear dependency of the band position of the LM on the AR has been in fact observed experimentally for metallic nanostructures of different morphologies that included nanorods (characterized by the plain cylinder and spherically capped cylinder) and ellipsoids [18, 23, 50, 67].

Upon increasing the AR of the nanobar, the absorption amplitude of the LM increases, and the maximum absorption occurs at $\mathrm{AR}=2.5$. Further increasing the length will result in a decrease of the absorption cross section. For the nanoparticle of small size, the absorption cross section is larger than the scattering one, so the extinction coefficient is mainly represented by the absorption properties. For larger nanoparticles (effective size of about $100 \mathrm{~nm}$ ) the scattering properties is dominant $[23,68,69]$. The theoretical observation for the nanocube and the nanobar here fits with these previous published data $[23,68]$. The absorption spectrum of nanoparticles which have two-fold symmetry like the plain cylinder, that is, the spherically capped cylinder and the spheroid, exhibits the excitation of both LM and TM. The absorption efficiency of the nanobar is now compared with that calculated for the other morphologies with the same AR. The way the polarization charges are distributed over the surface area of the nanostructure determines the position and intensity of the plasmonic band. At normal incidence, the TM band positions of the ellipsoid and capped cylinder are comparable. In the case of the nanobar and the plain cylinder, the band position is red-shifted as compared to the band of the ellipsoid as illustrated in Figure 6(a). It is clear that the nanobar has the maximum absorption because of the larger cross-sectional area. The ellipsoid and 


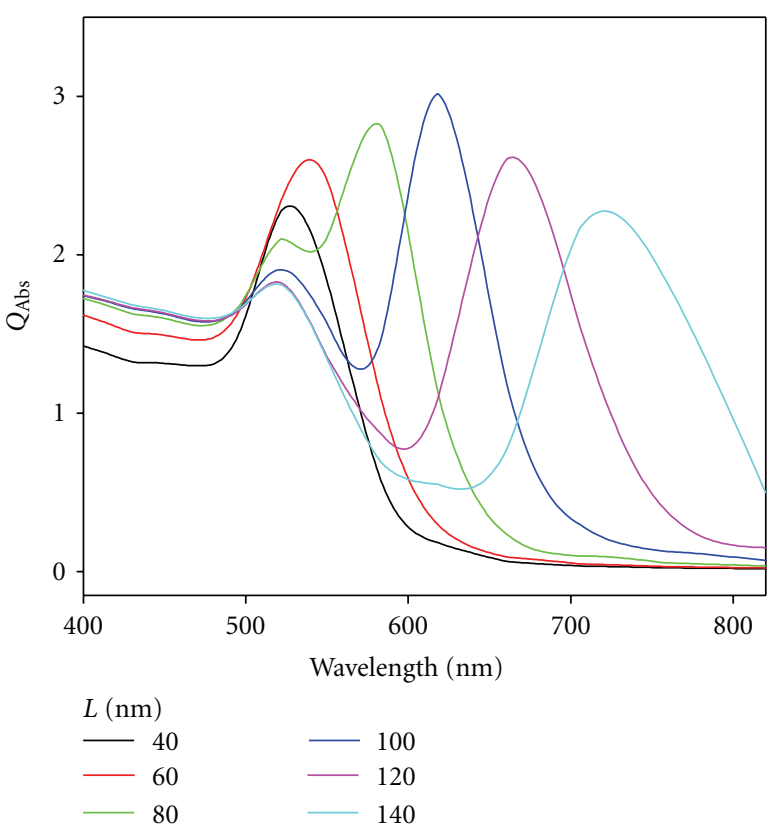

(a)

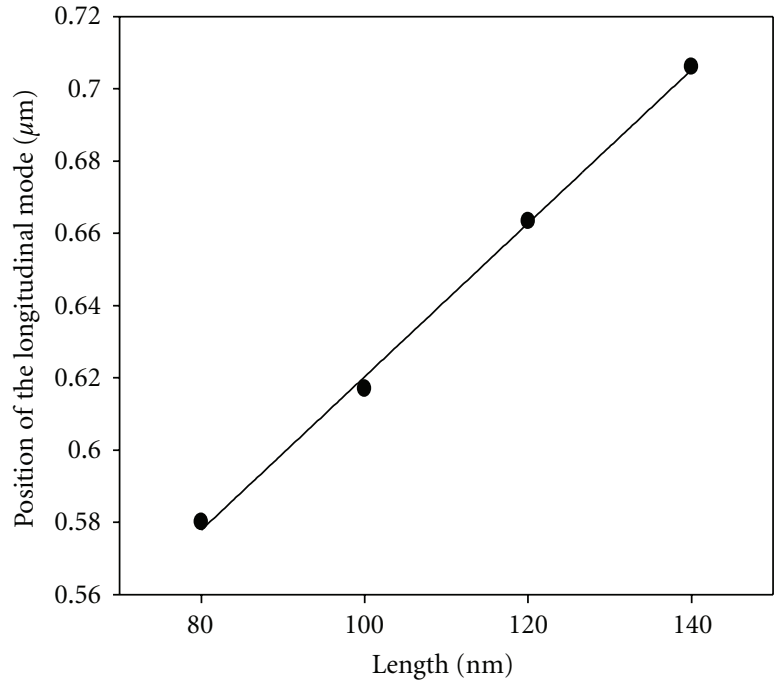

(b)

FIGURE 5: (a) Calculated absorption spectra of a nanobar of width of $40 \mathrm{~nm}$ and different aspect ratios at $\theta=30^{\circ}$, (b) position of the longitudinal band versus the nanobar length.

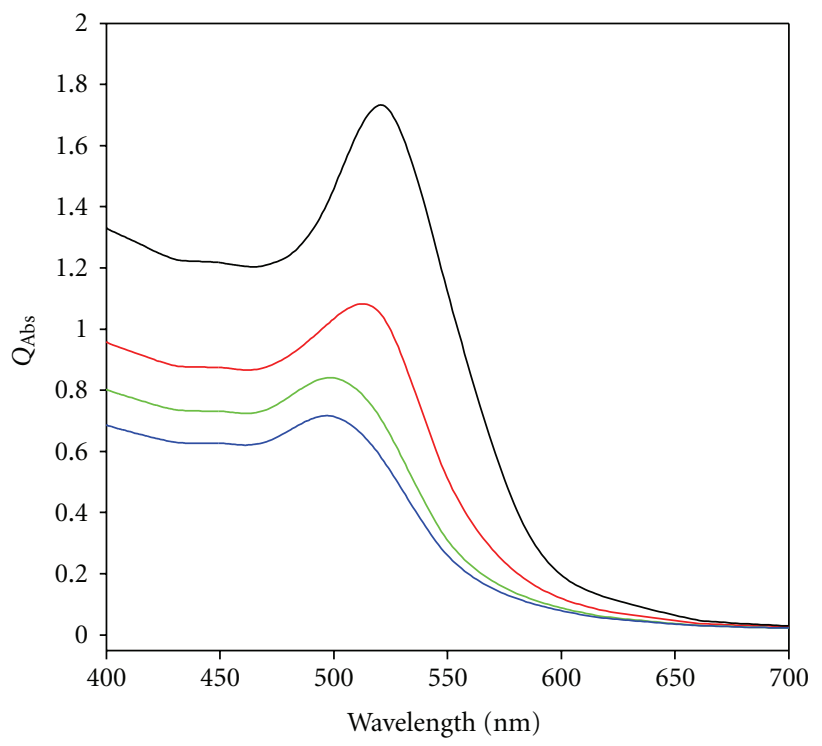

(a)

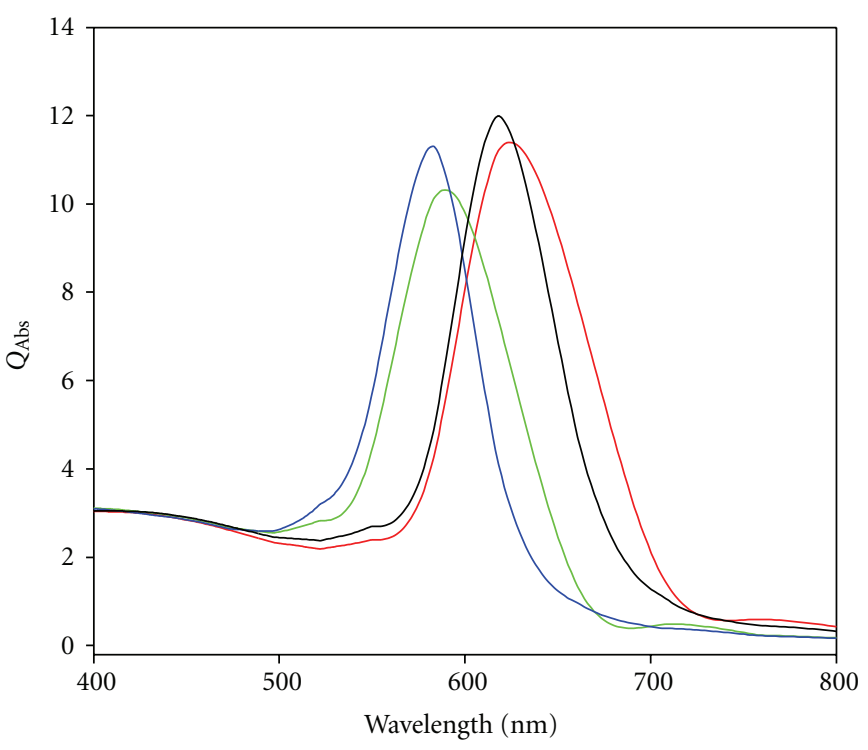

(b)

Figure 6: Comparison between the absorption spectra at (a) $\theta=0^{\circ}$ and (b) $\theta=90^{\circ}$ for a nanobar (black line), a plain cylinder (red line), an ellipsoid (blue line), and a capped spherical cylinder (green line).

the nanorod (the plain and the spherically capped) have a smaller width as compared to the nanobar, resulting in lesser absorption.

The calculated LM depends strongly on the morphology of the nanoparticle, and the redistribution of the polarization charges over a smooth flat area would result in a blue shift of the band position of the LM as in the case of the ellipsoid and the capped spherical cylinder with respect to other morphologies as shown in Figure 6(b).

\section{Conclusion}

The absorption efficiencies for a single gold nanocube and a single nanobar are modeled by using DDA. Results of 
the simulation of the optical properties of the nanocube show that the contribution to the extinction cross section comes mainly from the absorption one when the width $W \leq$ $80 \mathrm{~nm}$. When further increasing the width, the contribution of the scattering cross section becomes dominant. The position of its plasmonic band is red-shifted linearly with the side length. The distribution of the polarization on the corner of the nanocube increases the separation between the negative/positive charges, leading to a red shift in the band position of the LSPR mode as compared to a gold spherical particle of the same size. The absorption spectrum of a gold nanocube exhibits the excitation of single plasmonic band as compared to the excitation of several plasmonic bands in the case of a silver nanocube. Due to oscillations of the polarization charges parallel and perpendicular to the main axis of the nanobar, both the longitudinal and the transverse modes are observed. The intensity of the longitudinal mode is inversely proportional to the incident angle, while the intensity of the other mode is directly proportional to it. The band position of the most intense LSPR band can be tuned linearly in both the visible and near infrared regions by changing the length at a constant width. Distribution of the polarization charges across the nanoparticles of different morphologies (nanobar, cylinder, spherically capped cylinder, and ellipsoid) of the same aspect ratio results in a shift in the position for both bands. Considering the position of the ellipsoid plasmonic bands as a reference, both modes of the other shapes are red-shifted.

\section{Acknowledgments}

This work has been supported by the Natural Sciences and Engineering Research Council of Canada (NSERC) which is gratefully acknowledged. The authors also express their gratitude to B. T. Draine (Princeton University) and P. J. Flatau (University of California) for the free DDA code (DDSCAT 7.1).

\section{References}

[1] M. Faraday, "Experimental relations of gold (and other metals) to light," Philosophical Transactions of the Royal Society B, vol. 147, pp. 145-181, 1857.

[2] K. A. Willets and R. P. Van Duyne, "Localized surface plasmon resonance spectroscopy and sensing," Annual Review of Physical Chemistry, vol. 58, pp. 267-297, 2007.

[3] Y. Xia and N. J. Halas, "Shape-controlled synthesis and surface plasmonic properties of metallic nanoparticles," MRS Bulletin, vol. 30, pp. 338-348, 2005.

[4] D. J. Campbell and Y. Xia, "Plasmons: why should we care?" Journal of Chemical Education, vol. 84, no. 1, pp. 91-96, 2007.

[5] Y. Fang, "Optical absorption of nanoscale colloidal silver: aggregate band and adsorbate-silver surface band," Journal of Chemical Physics, vol. 108, no. 10, pp. 4315-4318, 1998.

[6] S. Link and M. A. El-Sayed, "Spectral properties and relaxation dynamics of surface plasmon electronic oscillations in gold and silver nanodots and nanorods," Journal of Physical Chemistry B, vol. 103, no. 40, pp. 8410-8426, 1999.

[7] C. Noguez, "Surface plasmons on metal nanoparticles: the influence of shape and physical environment," Journal of Physical Chemistry C, vol. 111, no. 10, pp. 3606-3619, 2007.
[8] J. Henzie, J. Lee, M. H. Lee, W. Hasan, and T. W. Odom, "Nanofabrication of plasmonic structures," Annual Review of Physical Chemistry, vol. 60, pp. 147-165, 2009.

[9] P. J. Kottman, O. J. F. Martin, D. R. Smith, and S. Schultz, "Plasmon resonances of silver nanowires with a nonregular cross section," Physical Review B, vol. 64, no. 23, Article ID 235402, pp. 1-10, 2001.

[10] H. Portalès, N. Pinna, and M. P. Pileni, "Optical response of ultrafine spherical silver nanoparticles arranged in hexagonal planar arrays studied by the DDA method," Journal of Physical Chemistry A, vol. 113, no. 16, pp. 4094-4099, 2009.

[11] S. Kim, Y. J. Jung, G. H. Gu, J. S. Suh, S. M. Park, and S. Ryu, "Discrete dipole approximation calculations of optical properties of silver nanorod arrays in porous anodic alumina," Journal of Physical Chemistry C, vol. 113, no. 37, pp. 1632116328, 2009.

[12] L. A. Sweatlock, S. A. Maier, H. A. Atwater, J. J. Penninkhof, and A. Polman, "Highly confined electromagnetic fields in arrays of strongly coupled Ag nanoparticles," Physical Review B, vol. 71, no. 23, Article ID 235408, pp. 1-7, 2005.

[13] P. Yang, H. Portalès, and M.-P. Pileni, "Ability to discern the splitting between longitudinal and transverse plasmon resonances in $\mathrm{Au}$ compared to Ag nanoparticles in close-packed planar arrays," Physical Review B, vol. 81, no. 20, Article ID 205405, 8 pages, 2010.

[14] R. Jin, Y. Cao, C. A. Mirkin, K. L. Kelly, G. C. Schatz, and J. G. Zheng, "Photoinduced conversion of silver nanospheres to nanoprisms," Science, vol. 294, no. 5548, pp. 1901-1903, 2001.

[15] M. A. El-Sayed, "Some interesting properties of metals confined in time and nanometer space of different shapes," Accounts of Chemical Research, vol. 34, no. 4, pp. 257-264, 2001.

[16] L. S. Slaughter, W. S. Chang, P. Swanglap et al., "Single-particle spectroscopy of gold nanorods beyond the quasi-static limit: varying the width at constant aspect ratio," Journal of Physical Chemistry C, vol. 114, no. 11, pp. 4934-4938, 2010.

[17] J. J. Penninkhof, A. Moroz, A. Van Blaaderen, and A. Polman, "Optical properties of spherical and oblate spheroidal gold shell colloids," Journal of Physical Chemistry C, vol. 112, no. 11, pp. 4146-4150, 2008.

[18] Y. Y. Yu, S. S. Chang, C. L. Lee, and C. R. C. Wang, "Gold nanorods: electrochemical synthesis and optical properties," Journal of Physical Chemistry B, vol. 101, no. 34, pp. 66616664, 1997.

[19] G. H. Chan, J. Zhao, E. M. Hicks, G. C. Schatz, and R. P. Van Duyne, "Plasmonic properties of copper nanoparticles fabricated by nanosphere lithography," Nano Letters, vol. 7, no. 7, pp. 1947-1952, 2007.

[20] C. Salzemann, I. Lisiecki, A. Brioude, J. Urban, and M. P. Pileni, "Collections of copper nanocrystals characterized by different sizes and shapes: optical response of these nanoobjects," Journal of Physical Chemistry B, vol. 108, no. 35, pp. 13242-13248, 2004.

[21] J. Aizpurua, G. W. Bryant, L. J. Richter, F. J. García De Abajo, B. K. Kelley, and T. Mallouk, "Optical properties of coupled metallic nanorods for field-enhanced spectroscopy," Physical Review B, vol. 71, no. 23, Article ID 235420, pp. 1-13, 2005.

[22] C. Ungureanu, R. G. Rayavarapu, S. Manohar, and T. G. Van Leeuwen, "Discrete dipole approximation simulations of gold nanorod optical properties: choice of input parameters and comparison with experiment," Journal of Applied Physics, vol. 105, no. 10, Article ID 102032, 2009.

[23] P. K. Jain, K. S. Lee, I. H. El-Sayed, and M. A. ElSayed, "Calculated absorption and scattering properties of 
gold nanoparticles of different size, shape, and composition: applications in biological imaging and biomedicine," Journal of Physical Chemistry B, vol. 110, no. 14, pp. 7238-7248, 2006.

[24] S. W. Prescott and P. Mulvaney, "Gold nanorod extinction spectra," Journal of Applied Physics, vol. 99, no. 12, Article ID 123504, 7 pages, 2006.

[25] B. N. Khlebtsov and N. G. Khlebtsov, "Multipole plasmons in metal nanorods: scaling properties and dependence on particle size, shape, orientation, and dielectric environment," Journal of Physical Chemistry C, vol. 111, no. 31, pp. 1151611527, 2007.

[26] A. M. Gobin, M. H. Lee, N. J. Halas, W. D. James, R. A. Drezek, and J. L. West, "Near-infrared resonant nanoshells for combined optical imaging and photothermal cancer therapy," Nano Letters, vol. 7, no. 7, pp. 1929-1934, 2007.

[27] X. Yang, S. E. Skrabalak, Z. Y. Li, Y. Xia, and L. V. Wang, "Photoacoustic tomography of a rat cerebral cortex in vivo with Au nanocages as an optical contrast agent," Nano Letters, vol. 7, no. 12, pp. 3798-3802, 2007.

[28] L. Tong, C. M. Cobley, J. Chen, Y. Xia, and J. X. Cheng, "Bright three-photon luminescence from gold/silver alloyed nanostructures for bioimaging with negligible photothermal toxicity," Angewandte Chemie-International Edition, vol. 49, no. 20, pp. 3485-3488, 2010.

[29] J. Chen, C. Glaus, R. Laforest et al., "Gold nanocages as photothermal transducers for cancer treatment," Small, vol. 6, no. 7, pp. 811-817, 2010.

[30] B. Nikoobakht, J. Wang, and M. A. El-Sayed, "Surface-enhanced Raman scattering of molecules adsorbed on gold nanorods: off-surface plasmon resonance condition," Chemical Physics Letters, vol. 366, no. 1-2, pp. 17-23, 2002.

[31] R. F. Aroca, R. A. Alvarez-Puebla, N. Pieczonka, S. SanchezCortez, and J. V. Garcia-Ramos, "Surface-enhanced Raman scattering on colloidal nanostructures," Advances in Colloid and Interface Science, vol. 116, no. 1-3, pp. 45-61, 2005.

[32] M. Moskovits, "Surface-enhanced Raman spectroscopy: a brief retrospective," Journal of Raman Spectroscopy, vol. 36, no. 6-7, pp. 485-496, 2005.

[33] J. N. Anker, W. P. Hall, O. Lyandres, N. C. Shah, J. Zhao, and R. P. Van Duyne, "Biosensing with plasmonic nanosensors," Nature Materials, vol. 7, no. 6, pp. 442-453, 2008.

[34] W. J. Galush, S. A. Shelby, M. J. Mulvihill, A. Tao, P. Yang, and J. T. Groves, "A nanocube plasmonic sensor for molecular binding on membrane surfaces," Nano Letters, vol. 9, no. 5, pp. 2077-2082, 2009.

[35] A. J. Haes and R. P. Van Duyne, "A nanoscale optical biosensor: sensitivity and selectivity of an approach based on the localized surface plasmon resonance spectroscopy of triangular silver nanoparticles," Journal of the American Chemical Society, vol. 124, no. 35, pp. 10596-10604, 2002.

[36] S. Lal, S. E. Clare, and N. J. Halas, "Nanoshell-enabled photothermal cancer therapy: impending clinical impact," Accounts of Chemical Research, vol. 41, no. 12, pp. 1842-1851, 2008.

[37] D. P. Fromm, A. Sundaramurthy, P. James Schuck, G. Kino, and W. E. Moerner, "Gap-dependent optical coupling of single "bowtie" nanoantennas resonant in the visible," Nano Letters, vol. 4, no. 5, pp. 957-961, 2004.

[38] V. E. Ferry, M. A. Verschuuren, H. B. T. Li et al., "Light trapping in ultrathin plasmonic solar cells," Optics Express, vol. 18, no. 13, pp. A237-A245, 2010.

[39] N. Chandrasekharan and P. Y. Kainat, "Improving the photoelectrochemical performance of nanostructured $\mathrm{TiO}_{2}$ films by adsorption of gold nanoparticles," Journal of Physical Chemistry B, vol. 104, no. 46, pp. 10851-10857, 2000.
[40] V. E. Ferry, L. A. Sweatlock, D. Pacifici, and H. A. Atwater, "Plasmonic nanostructure design for efficient light coupling into solar cells," Nano Letters, vol. 8, no. 12, pp. 4391-4397, 2008.

[41] E. Hao, G. C. Schatz, and J. T. Hupp, "Synthesis and optical properties of anisotropic metal nanoparticles," Journal of Fluorescence, vol. 14, no. 4, pp. 331-341, 2004.

[42] E. Hao and G. C. Schatz, "Electromagnetic fields around silver nanoparticles and dimers," Journal of Chemical Physics, vol. 120, no. 1, pp. 357-366, 2004.

[43] I. O. Sosa, C. Noguez, and R. G. Barrera, "Optical properties of metal nanoparticles with arbitrary shapes," Journal of Physical Chemistry B, vol. 107, no. 26, pp. 6269-6275, 2003.

[44] B. T. Draine, "The discrete-dipole approximation and its application to interstellar graphite grains," Astrophysical Journal, vol. 333, pp. 848-872, 1988.

[45] B. T. Draine and P. J. Flatau, "Discrete-dipole approximation for scattering calculations," Journal of the Optical Society of America A, vol. 11, no. 4, pp. 1491-1499, 1994.

[46] B. T. Draine and J. Goodman, "Beyond Clausius-Mossotti: wave propagation on a polarizable point lattice and the discrete dipole approximation," Astrophysical Journal, vol. 405, no. 2, pp. 685-697, 1993.

[47] B. T. Draine and P. J. Flatau, "Diserete-dipole approximation for periodic targets: theory and tests," Journal of the Optical Society of America A, vol. 25, no. 11, pp. 2693-2703, 2008.

[48] M. J. Collinge and B. T. Draine, "Discrete-dipole approximation with polarizabilities that account for both finite wavelength and target geometry," Journal of the Optical Society of America A, vol. 21, no. 10, pp. 2023-2028, 2004.

[49] J. J. Goodman, B. T. Draine, and P. J. Flatau, "Application of fast-Fourier-transform techniques to the discrete-dipole approximation," Optics Letters, vol. 16, no. 15, pp. 1198-1200, 1991.

[50] A. Brioude, X. C. Jiang, and M. P. Pileni, "Optical properties of gold nanorods: DDA simulations supported by experiments," Journal of Physical Chemistry B, vol. 109, no. 27, pp. 1313813142, 2005.

[51] J. Zhu, C. Kan, X. Zhu et al., "Synthesis of perfect silver nanocubes by a simple polyol process," Journal of Materials Research, vol. 22, no. 6, pp. 1479-1485, 2007.

[52] B. J. Wiley, S. H. Im, Z. Y. Li, J. McLellan, A. Siekkinen, and Y. Xia, "Maneuvering the surface plasmon resonance of silver nanostructures through shape-controlled synthesis," Journal of Physical Chemistry B, vol. 110, no. 32, pp. 15666-15675, 2006.

[53] K. Young, "Synthesis and galvanic replacement reaction of silver nanocubes in organic medium," in Materials, Nnin Reu Research Accomplishments, 2006.

[54] A. R. Siekkinen, J. M. McLellan, J. Chen, and Y. Xia, "Rapid synthesis of small silver nanocubes by mediating polyol reduction with a trace amount of sodium sulfide or sodium hydrosulfide," Chemical Physics Letters, vol. 432, no. 4-6, pp. 491496, 2006.

[55] Y. T. Lee, S. H. Im, B. Wiley, and Y. Xia, "Quick formation of single-crystal nanocubes of silver through dual functions of hydrogen gas in polyol synthesis," Chemical Physics Letters, vol. 411, no. 4-6, pp. 479-483, 2005.

[56] B. N. Khlebtsov, V. A. Khanadeev, I. L. Maksimova, G. S. Terentyuk, and N. G. Khlebtsov, "Silver nanocubes and gold nanocages: fabrication and optical and photothermal properties," Nanotechnologies in Russia, vol. 5, no. 7-8, pp. 454-468, 2010. 
[57] B. J. Wiley, Y. Chen, J. M. McLellan et al., "Synthesis and optical properties of silver nanobars and nanorice," Nano Letters, vol. 7, no. 4, pp. 1032-1036, 2007.

[58] H. Chen, Z. Sun, W. Ni et al., "Plasmon coupling in clusters composed of two-dimensionally ordered gold nanocubes," Small, vol. 5, no. 18, pp. 2111-2119, 2009.

[59] D. Yu and V. W. W. Yam, "Controlled synthesis of monodisperse silver nanocubes in water," Journal of the American Chemical Society, vol. 126, no. 41, pp. 13200-13201, 2004.

[60] G. Mie, "Beitrage zur optik truber medien, speziell kolloida ler metallosungen," Annalen der Physik, vol. 25, p. 377, 1908.

[61] R. Gans, "Über die Form ultramikroskopischer Silberteilchen," Annalen der Physik, vol. 352, no. 10, pp. 270-284, 1915.

[62] P. B. Johnson and R. W. Christy, "Optical constants of the noble metals," Physical Review B, vol. 6, no. 12, pp. 4370-4379, 1972.

[63] Q. Zhang, W. Li, L. P. Wen, J. Chen, and Y. Xia, "Facile synthesis of $\mathrm{Ag}$ nanocubes of 30 to $70 \mathrm{~nm}$ in edge length with $\mathrm{CF}_{3} \mathrm{COOAg}$ as a precursor," Chemistry - A European Journal, vol. 16, no. 33, pp. 10234-10239, 2010.

[64] X. Wu, T. Ming, X. Wang, P. Wang, J. Wang, and J. Chen, "High-photoluminescence-yield gold nanocubes: for cell imaging and photothermal therapy," ACS Nano, vol. 4, no. 1, pp. 113-120, 2010.

[65] J. J. Mock, M. Barbic, D. R. Smith, D. A. Schultz, and S. Schultz, "Shape effects in plasmon resonance of individual colloidal silver nanoparticles," Journal of Chemical Physics, vol. 116, no. 15, pp. 6755-6759, 2002.

[66] H. A. Atwater and A. Polman, "Plasmonics for improved photovoltaic devices," Nature Materials, vol. 9, no. 3, pp. 205-213, 2010.

[67] E. Stefan Kooij and B. Poelsema, "Shape and size effects in the optical properties of metallic nanorods," Physical Chemistry Chemical Physics, vol. 8, no. 28, pp. 3349-3357, 2006.

[68] D. Derkacs, S. H. Lim, P. Matheu, W. Mar, and E. T. Yu, "Improved performance of amorphous silicon solar cells via scattering from surface plasmon polaritons in nearby metallic nanoparticles," Applied Physics Letters, vol. 89, no. 9, Article ID 093103, 2006.

[69] D. M. Schaadt, B. Feng, and E. T. Yu, "Enhanced semiconductor optical absorption via surface plasmon excitation in metal nanoparticles," Applied Physics Letters, vol. 86, no. 6, Article ID 063106, pp. 1-3, 2005. 

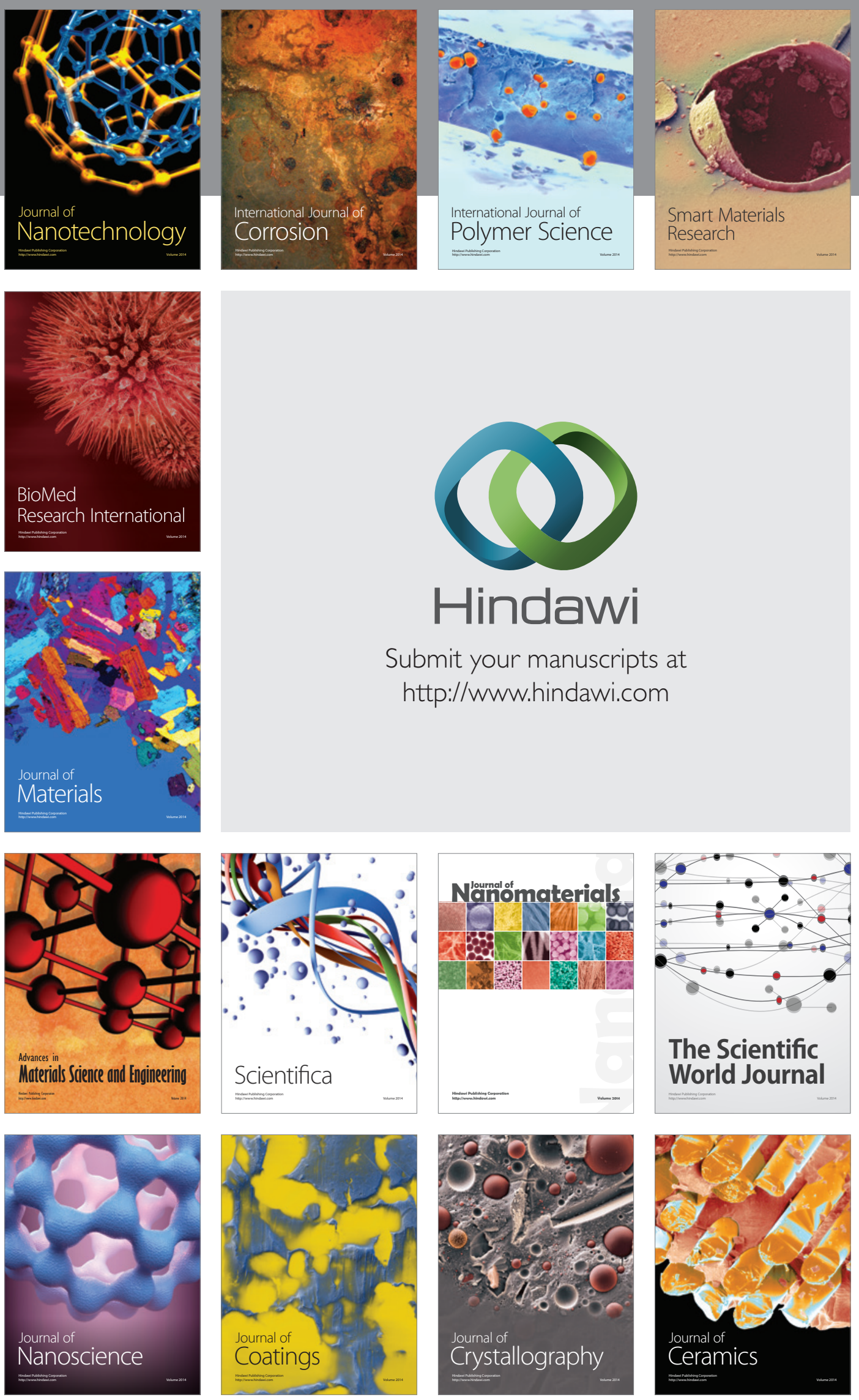

The Scientific World Journal

Submit your manuscripts at

http://www.hindawi.com

\section{World Journal}

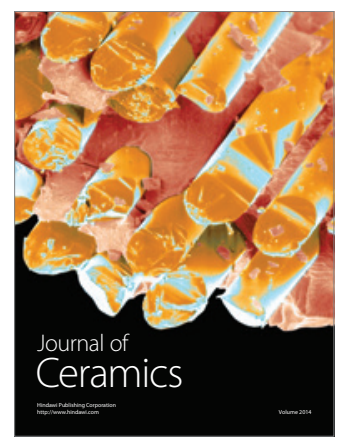

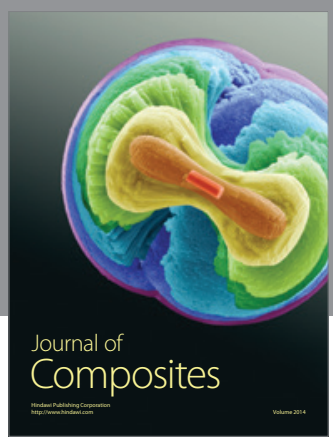
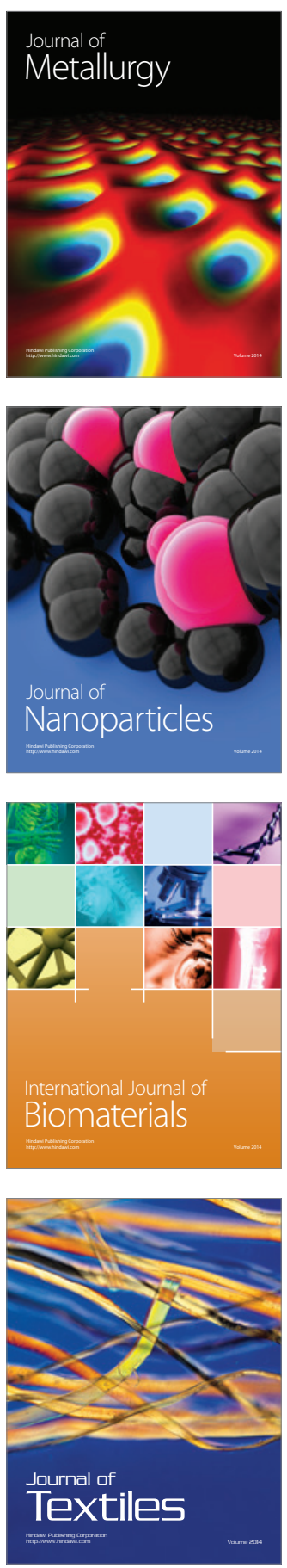